\title{
Die globale Informationsgesellschaft: Visionen, Bevölkerung und Macht
}

Cees J. Hamelink

\section{CpenEdition}

\section{Journals}

Electronic version

URL: http://journals.openedition.org/sjep/576

DOI: $10.4000 /$ sjep. 576

ISSN: 1663-9677

\section{Publisher}

Institut de hautes études internationales et du développement

\section{Printed version}

Date of publication: 1 novembre 2003

Number of pages: 165-189

ISSN: 1660-5926

\section{Electronic reference}

Cees J. Hamelink, « Die globale Informationsgesellschaft: Visionen, Bevölkerung und Macht », Schweizerisches Jahrbuch für Entwicklungspolitik [Online], 22-2 | 2003, Online erschienen am: 14 Juni 2010, abgerufen am 08 September 2020. URL : http://journals.openedition.org/sjep/576 ; DOI : https://doi.org/10.4000/sjep.576 


\title{
Die globale Informationsgesellschaft: Visionen, Bevölkerung und Macht
}

\author{
Cees J. Hamelink*
}

D ieses Kapitel analysiert die in zahlreichen vorbereitenden Texten zum Weltgipfel über die Informationsgesellschaft (WSIS) gebotenen Visionen. Dabei wird deutlich, dass diese mehrheitlich von technologischem Optimismus geprägt sind. Besonders auffallend an diesen Texten ist das grosse Gewicht, das der „Information“ beigemessen wird, während die „,Kommunikation" kaum je erwähnt wird. Dies überrascht, denn die wichtigste Voraussetzung für die Errichtung einer einschliessenden globalen Gesellschaft und für das Empowerment ist die Gewährleistung der Kommunikation zwischen den Menschen.

Ferner lassen die meisten vorbereitenden Dokumente zum WSIS einen akuten Mangel an grundlegender gesellschaftlicher Analyse erkennen, was dazu führt, dass die gebotenen Aussagen mehrheitlich unbegründet bleiben und übereilt erscheinen. Ein Paradebeispiel dafür ist die Loslösung der so genannten „,digitalen Kluft" von der umfassenden Problematik der Entwicklungsunterschiede. Das Kapitel bietet eine kritische Betrachtung der geltenden Regelungen im Telekommunikationsbereich und auf dem Gebiet der Rechte am geistigen Eigentum, beschreibt die wichtigsten weltpolitischen Verschiebungen in Bezug auf Information und Kommunikation und plädiert für eine auf Bürgerbeteiligung und Menschenrechten beruhende Vision für den WSIS. Diese Vision setzt die Schaffung eines Forums voraus, dessen Mitglieder sich entschlossen für die Errichtung einer Informationsgesellschaft von der Basis her engagieren. Das Kapitel schliesst mit einer Liste von Empfehlungen für dieses Forum.

Der demnächst stattfindende Weltgipfel über die Informationsgesellschaft (World Summit on the Information Society, WSIS) gründet auf einem umstrittenen Konzept, nämlich demjenigen der Informationsgesellschaft. Eine allgemein akzeptierte Definition des Begriffs „Informationsgesellschaft“" existiert nicht, und seine Bedeutung ist verschiedentlich ernsthaft hinterfragt worden: Während von Wissenschaftlern gar behauptet wurde, der Begriff stehe in keinem Zusammenhang mit den heutigen gesellschaftlichen Gegebenheiten, wurde von anderer Seite die Frage aufgeworfen, ob Gesellschaften anhand einer einzigen Variablen überhaupt adäquat beschrieben werden können, und falls ja, ob die Information dabei eine präzisere Kategorie darstelle als beispielsweise Reichtum, Kriminalität oder Aggression. Tatsache ist jedenfalls, dass Gesellschaften sich auf weitgehend unterschiedlichen Achsen entwickeln, und dass, falls auf den Hinweis auf die Information nicht verzichtet werden kann, vorzugsweise

* Professor für internationale Kommunikation an der Universität Amsterdam, Niederlande. 
von „den Informationsgesellschaften“ gesprochen werden sollte. Denn je nach Standpunkt wird der Begriff „Informationsgesellschaft" mit unterschiedlichen Dingen in Zusammenhang gebracht: mehr Telefone, mehr Geld, stärkere Regulierung, mehr Empowerment.

Bedauerlicherweise wird in der vorbereitenden Dokumentation zum Gipfel das Konzept der Informationsgesellschaft ohne kritische Hinterfragung verwendet. Ein Beispiel für häufig verwendete Phrasen findet sich etwa im Beitrag der EU zur PrepCom 2 (die im Februar 2003 in Genf tagte), welcher ausführt, dass die Informationsgesellschaft ein bedeutendes Potenzial zur Förderung der nachhaltigen Entwicklung, der Demokratie, der Transparenz, der Ergebnisverantwortung und der guten Regierungsführung besitzt (Übersetzung des englischen Originaltextes). Dass eine seriöse Organisation davon auszugehen scheint, dass etwas nicht näher Definiertes (nämlich die Informationsgesellschaft) potenziell etwas bewirken kann, ist befremdend. Immerhin wird in einigen vorbereitenden Texten versucht, den Begriff der Informationsgesellschaft zu umschreiben.

Die Bavaro-Erklärung der lateinamerikanischen und karibischen Regionalkonferenz (29. bis 31. Januar 2003) hält fest, dass es sich bei der Informationsgesellschaft um ein wirtschaftliches und soziales System handelt, bei dem Wissen und Information die grundlegenden Pfeiler für Wohlstand und Fortschritt darstellen. Die Tokio-Erklärung der asiatischen Regionalkonferenz (13. bis 15. Januar 2003) wiederum beschreibt die Informationsgesellschaft als Gesellschaftsform, in der hoch entwickelte IKT-Netzwerke, ein gerechter und umfassender Zugang zu Information, geeignete Inhalte in allgemein zugänglichen Formaten und eine wirksame Kommunikation die Menschen zur Ausschöpfung ihres Potenzials befähigen, die wirtschaftliche und soziale Entwicklung fördern, die Lebensqualität für alle Menschen verbessern, Armut und Hunger lindern und Entscheidungsprozesse erleichtern.

\section{Von Zuversicht und Euphorie geprägte Visionen}

Die Mehrheit der von den verschiedenen Anspruchsgruppen skizzierten Visionen der Informationsgesellschaft stimmen zuversichtlich, ja euphorisch. Die zur Beschreibung der Visionen verwendeten Schlüsselbegriffe ähneln sich weitgehend: Die Informationsgesellschaft sollte einschliessend sein und die Beteiligung sowie den Zugang der grösstmöglichen Zahl von Menschen gewährleisten. Die Informationsgesellschaft sollte zum Empowerment der Menschen beitragen, ein vorteilhaftes Umfeld schaffen und den Kapazitätsaufbau unterstützen. Die Informationsgesellschaft sollte auf demokratischen Grundlagen ruhen. Die Hauptziele der Informationsgesellschaft sind die nachhaltige Entwicklung, die kulturelle Vielfalt sowie die Gleichstellung der Geschlechter.

Ähnliche Aussagen finden sich auch im Entwurf der Abschlusserklärung (Version vom 27. Februar 2003):

$\square$ Die Ausschöpfung des Potenzials der Informations- und Kommunikationstechnologien (IKT) kann dazu beitragen, neue und bessere Massnahmen zur Verringerung der Armut und zur Gewährleistung der Gleichheit und sozialen Gerechtigkeit zu finden. 
$\square \quad$ Wissen und Information stellen eine der wichtigsten Quellen für Wohlstand und Fortschritt dar.

$\square$ Das ungenutzte Potenzial der IKT zur Steigerung von Produktivität und Lebensqualität ist von zentraler Bedeutung.

$\square$ Die Vorzüge der Informationsgesellschaft sollten allen Menschen zugute kommen und die Entwicklung begünstigen.

$\checkmark$ Beim Aufbau einer Informationsgesellschaft ist sicherzustellen, dass auch Frauen den zunehmenden Einsatz von IKT als Chance zum Empowerment nutzen und umfassend an der Gestaltung der politischen, wirtschaftlichen und sozialen Entwicklung beteiligt sind.

$\square$ Die kulturelle und sprachliche Vielfalt ist zu fördern, wobei das Wissen und die Fähigkeiten autochthoner Völker einen wesentlichen Beitrag leisten können.

$\square$ Ziel der Informationsgesellschaft muss es sein, bestehende sozioökonomische Unterschiede in den Gesellschaften abzubauen.

$\square$ Ein gleichberechtigter und angemessener Zugang für alle Menschen ist ein wesentliches Element einer gut entwickelten, finanzierbaren und leicht zugänglichen Informations- und Kommunikationsinfrastruktur.

$\square$ Als Massnahme zur Linderung der Armut sind benachteiligten gesellschaftlichen Gruppen, insbesondere jenen der unteren Einkommensschichten, Informations- und Kommunikationsdienstleistungen zur Verfügung zu stellen.

$\square$ Um die aktive Teilnahme an der Informationsgesellschaft, das Verständnis ihres Wesens und die weitestgehende Nutzung der entstehenden Möglichkeiten zu gewährleisten, sollten die Menschen befähigt werden, sich die erforderlichen Kenntnisse anzueignen.

$\square$ Um die wirtschaftlichen und sozialen Vorteile der Informationsgesellschaft zu maximieren, müssen die Regierungen ein vertrauenswürdiges, transparentes und nicht diskriminierendes gesetzliches, regulatorisches und politisches Umfeld schaffen.

$\square$ Die Informationsgesellschaft gründet auf dem Respekt vor der Kultur und vor den Ausdrucksformen des Kulturschaffens.

$\square$ Die Mehrsprachigkeit ist zu fördern und die kulturelle Vielfalt zu erhalten, denn sie sind die treibende Kraft für die Entwicklung von Inhalten, die auf lokaler und internationaler Ebene genutzt werden.

$\square$ Die umfassende und effektive Einbindung aller Anspruchsgruppen ist eine grundlegende Voraussetzung für die Entwicklung neuer IKT-Anwendungen.

$\square$ Die Völkergemeinschaft ist gefordert, die technische und finanzielle Zusammenarbeit auf multilateraler und bilateraler Ebene sicherzustellen.

Allgemein wird davon ausgegangen, dass die Informationsgesellschaft eine noch nie da gewesene Win-Win-Situation hervorbringen und die Lebensbedingungen der gesamten Menschheit verbessern kann. Die meisten am WSIS-Prozess beteiligten Anspruchsgruppen scheinen der vom Vorsitzenden des WSIS-Vorbereitungsausschusses, Adama Samassekou, geäusserten Sichtweise beizupflichten, 
welche in einem Entwurf für ein Orientierungspapier zur PrepCom 2 vom vergangenen Februar in Genf festgehalten wurde. Samassekou definierte eine einschliessende globale Informationsgesellschaft als eine Gesellschaftsform, in der alle Menschen ohne Unterschied befähigt werden, Information und Wissen für ihre wirtschaftliche, soziale, kulturelle und politische Entwicklung frei zur Verfügung zu stellen, zu erhalten, zu teilen und zu nutzen.

Obwohl diese Intentionen zweifellos zu begrüssen sind, gilt es zu bedenken, dass sie als Visionen vorgeschlagen werden, und dass es keinen empirisch fundierten Beweis für ein derartiges Potenzial der Informationsgesellschaft gibt.

\section{Techno-Optimismus}

Die überwiegende Zahl der vorbereitenden Texte bieten einen positiven, wenn nicht gar euphorischen Ausblick auf das Potenzial der Informations- und Kommunikationstechnologien. Häufig wird darauf hingewiesen, dass die IKT im Entwicklungsbereich von grossem Nutzen sein werden. Auffallend an den meisten Visionen ist der technologische Optimismus. Dieser ist aber äusserst problematisch, denn er impliziert die Weigerung, sich ernsthaft mit negativen Szenarien für die künftige Entwicklung auseinander zu setzen. Wie alle technologischen Errungenschaften haben auch die IKT Nachteile. Es wäre deshalb nur vernünftig und ein Zeichen von Verantwortungsbewusstsein, sich auf ein „Worst CaseSzenario" vorzubereiten.

Das Konzept der Informationsgesellschaft ist eng mit der derzeitigen technologisch geprägten Kultur verknüpft. Diese vorherrschende Art der Interaktion zwischen Gesellschaft und Technologie ist weitgehend von Irrationalität und Verantwortungslosigkeit geprägt und lässt sich mit Hilfe von drei Metaphern zusammenfassen: die Titanic, Kassandra und Dr. Frankenstein.

- Die Titanic symbolisiert ein schier grenzenloses Vertrauen in die technologische Perfektion: Da das Schiff unsinkbar ist, kann auf eine genügende Anzahl Rettungsboote verzichtet werden. Die vorhandenen Risiken technologischer Innovationen werden somit nicht ernst genommen. Die moderne technologisch geprägte Kultur weist eine starke Tendenz hin zu einer risikofreien Gesellschaft auf. Allerdings wird das Streben nach einer risikolosen Steuerung gesellschaftlicher Prozesse durch die Unberechenbarkeit und die Unbeständigkeit der Menschheit weitgehend zunichte gemacht. Der Mensch wird zunehmend als der eigentliche Risikofaktor wahrgenommen. Angesichts dessen versucht die moderne Gesellschaft auf verschiedene Weise, dieses Risiko einzudämmen, etwa durch die umfassende Kontrolle des menschlichen Verhaltens durch die allgegenwärtige Videoüberwachung und durch die elektronische Registrierung der Mobilität der Menschen. Der Einsatz humanoider Roboter an Stelle von Menschen wäre der nächste logische Schritt in diesem Prozess.

- Kassandra ist die Tochter des trojanischen Königs Priamus, welche die Trojaner vor den im hölzernen Pferd versteckten Griechen warnte. Sie besass die Gabe, die Zukunft voraussehen zu können, und wurde von Apollo aus Rache damit bestraft, dass niemand ihren Prophezeiungen Glauben schenkte. 
Auch dies ist ein charakteristischer Zug der technologisch geprägten Kultur: Warnende Stimmen werden ignoriert. Wenn Entscheidungsträger sich am Anfang einer neuen Ära wähnen und Siegesgewissheit, Zeit- und Wettbewerbsdruck vorherrschen, dann werden Warnsignale missachtet, Dissidenten zum Schweigen gebracht und technologische Entscheidungen gewissermassen im Blindflug getroffen.

- Dr. Frankenstein ist eine Figur aus dem gleichnamigen Roman von Mary Shelley (1818). Er flieht vor dem Monster, das er in seinem Labor herangezüchtet hat, aber das Monster verfolgt ihn und zwingt ihn, die Verantwortung für seine Schöpfung wahrzunehmen. Diese Metapher veranschaulicht die kritische Frage nach der Rechenschaft für die Folgen technologischer Innovationen. Wer ist schuld, wenn etwas schief geht? Wer übernimmt die Verantwortung für die lebensbedrohlichen Umweltprobleme, die durch die Überwindung der „digitalen Kluft“ entstehen könnten, wie beispielsweise der enorme weltweite Energieverbrauch, die bedeutenden $\mathrm{CO}_{2}$-Emissionen von Druckern und Computern und der Elektroschrott, dessen Volumen durch die Kurzlebigkeit von Mobiltelefonen und Hardware in die Höhe schnellen dürfte?

". UN MONDE PLUS JUSTE,..

\section{... LUTTER CONTRE LA PAUVRETE...}

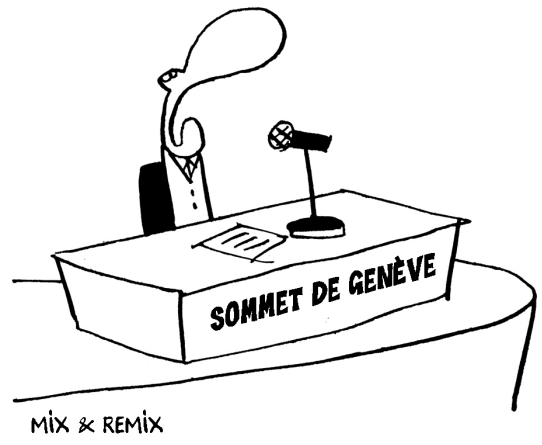

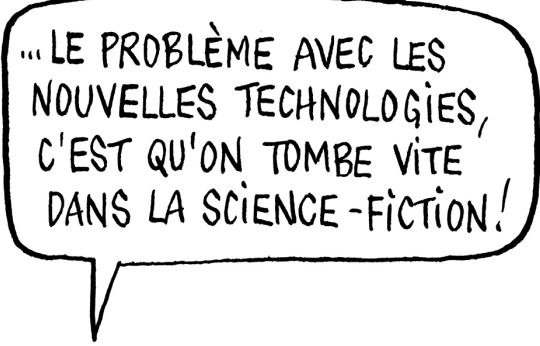


Eine gerechtere Welt, Kampf gegen die Armut...

Das Problem mit den neuen Technologien ist nur, dass man schnell in die Science-fiction abrutscht! 
„Information“ ist offensichtlich von zentraler Bedeutung für die Informationsgesellschaft. Überlegungen zur Zukunft der Informationsgesellschaften gründen häufig auf einer Reihe von Gemeinplätzen: Mehr Information ist besser als weniger; mehr Information schafft mehr Wissen und Verständnis; der ungehinderte Informationsfluss trägt zur Konfliktverhinderung bei; mehr Information ist gleichbedeutend mit weniger Ungewissheit und adäquateren Wahlmöglichkeiten; angemessen informierte Menschen verhalten sich entsprechend; mehr Information führt zu mehr Macht; und Menschen, die mehr von ihren Mitmenschen wissen, verstehen diese besser und sind weniger konfliktbereit. So attraktiv diese Behauptungen auch erscheinen mögen, sie treffen längst nicht immer zu.

Um die dringlichsten Probleme auf der Welt zu lösen, benötigen die Menschen nicht unbedingt mehr Information und Wissen. Vielmehr müssen sie die Fähigkeit erwerben, über kulturelle, religiöse und sprachliche Grenzen hinweg miteinander in einen Dialog zu treten. Dieser Dialog ist für den Kontakt zwischen den einzelnen Zivilisationen von entscheidender Bedeutung. Überraschenderweise wird die Kommunikation in den meisten vorbereitenden Papieren zum Weltgipfel über die Informationsgesellschaft kaum mehr erwähnt. Damit besteht die Gefahr, dass bei diesem Gipfel derselbe Fehler gemacht wird wie bei der UN-Menschenrechtskonferenz (Wien, 1993), in deren Abschlusserklärung nicht auf die Kommunikation verwiesen wird, sondern lediglich Information und Nachrichten genannt werden. Dabei lautet doch die zentrale Frage, wie „Kommunikationsgesellschaften“ zu gestalten sind. Die Welt braucht nicht mehr Informationsverarbeitung, sondern Kommunikationsfähigkeit. Ironischerweise nimmt nämlich unsere Kommunikations- und Dialogfähigkeit in gleichem Masse $a b$, in dem unsere Kapazitäten zur Verarbeitung und Verbreitung von Information und Wissen zunehmen.

In komplexen modernen Gesellschaften sind die Menschen gefordert, miteinander zu kommunizieren. Bei der Bewältigung der dringlichsten sozialen Probleme ist die Kommunikationsfähigkeit weitaus bedeutender als die Fähigkeit, Informationen zu verbreiten. Es ist alarmierend, dass die Menschheit in der Lage ist, Informations- oder gar Kommunikationsgesellschaften zu errichten, deren Mitglieder nicht fähig sind, miteinander zu reden. Dass die Kommunikation nirgendwo erwähnt wird, ist eine ernst zu nehmende Unterlassung und entspricht der vereinfachenden Sichtweise, dass die Schaffung, der Empfang, das Teilen und Nutzen von Information und Wissen die Entwicklung begünstigen, und zwar ungeachtet dessen, ob die Menschen fähig, beziehungsweise in der Lage sind, miteinander zu kommunizieren.

Irritierenderweise deuten jene Dokumente, in denen die Kommunikation erwähnt wird, darauf hin, dass der grundlegende Unterschied zwischen Information und Wissen auf der einen, und Kommunikation auf der anderen Seite ausser Acht gelassen wird. Nicht selten entsteht der Eindruck, dass Kommunikation mit der Verbreitung von Ideen und Informationen gleichgesetzt wird. Dies ist insofern verständlich, als auch Massenmedien häufig als Kommunikationsmittel bezeichnet werden. Obgleich dies nicht a priori falsch ist, nehmen die Massen- 
medien in den meisten Fällen doch eine Verbreitungsfunktion wahr. Die Information und das Wissen, das sie verbreiten, kann zwar zu Interaktionen führen, aber dies ist nicht oft der Fall.

Die Kommunikation ist eine der wichtigsten Voraussetzungen für die Schaffung einer einschliessenden globalen Gesellschaft und Mittel der Menschen zur wirtschaftlichen, sozialen, kulturellen und politischen Entwicklung. Außer den Positionen der CRIS-Kampagne (Communication Rights in the Information Society) wird die Vision einer einschliessenden globalen Kommunikationsgesellschaft an keiner Stelle erwähnt. Die Schlusserklärung der asiatischen Nichtregierungsorganisation (Bangkok, 22. bis 24. November 2002) führt in ihren Grundsätzen Folgendes aus: Kommunikationsrechte sind eine grundlegende Voraussetzung für die Demokratie und die Entwicklung der Menschheit, auf die an verschiedenen Stellen, beispielsweise in Artikel 19 der Allgemeinen Erklärung der Menschenrechte verwiesen wird. Diese Feststellung zeugt von einem erheblichen Missverständnis, denn Artikel 19 der Erklärung der Menschenrechte handelt nicht von den Kommunikationsrechten, sondern von der Meinungsfreiheit und der freien Meinungsäusserung. Die Kommunikation wird darin nicht erwähnt. In der Tat unterscheidet sich der Kommunikationsprozess grundlegend von der Äusserung und Verbreitung von Informationen und Meinungen. Bis heute gibt es kein anerkanntes Recht auf ein Umfeld, welches die Kommunikation zwischen den Menschen ermöglicht.

\section{Keine kritische gesellschaftliche Analyse}

So unbefriedigend das Konzept der Informationsgesellschaft aus intellektueller Sicht auch sein mag, so bietet es doch gewisse analytische Vorteile. Der Hinweis auf die Gesellschaft erinnert an bekannte soziologische Fragestellungen nach Macht, Nutzen und Partizipation: Wer hat den grössten Nutzen, wer entscheidet, wer ist beteiligt und wer trägt die Verantwortung ? Aber genau diese Analyse wird in den meisten vorbereitenden Texten umgangen. Dies ist umso störender, als die lobenswerten Visionen darüber, was die Informationsgesellschaft sei oder sein sollte, bei den internationalen Bemühungen um eine bessere Welt einen festen Stellenwert einnehmen. Zahlreich sind die Schlagwörter aus vergangenen Jahrzehnten: Demokratie, Vielfalt, Befähigung, Beteiligung, Gleichstellung der Geschlechter und Überwindung von Unterschieden. In der Tat ein inspirierender Sprachgebrauch! Offen bleibt jedoch die Frage, weshalb die internationale Gemeinschaft bis heute keine ernsthaften Bemühungen zur Umsetzung dieser Anliegen unternommen hat. Weshalb war die Völkergemeinschaft in den vergangenen Jahrzehnten nicht gewillt, ihren Worten auch Taten folgen zu lassen?

Bevor übereilte Aussagen über das Potenzial der Informationsgesellschaft, der IKT, von Information und Wissen gemacht werden, ist zu klären, weshalb die Welt bis heute keine einschliessende Gemeinschaft (inclusive community) hervorgebracht hat, weshalb noch keine nachhaltige Entwicklung stattgefunden hat, weshalb es bis heute keine transparente und verantwortungsvolle globale Regierungsführung gibt und weshalb die Menschen nicht gleichberechtigt in ihre 
jeweilige Gesellschaft eingebunden sind. Die Regierungsführung in der Informationsgesellschaft sollte unbedingt vertieft erörtert werden. Zugegeben, die Rhetorik zeichnet mit Schlagwörtern wie Multi-Stakeholder, Partizipation, Demokratie und Transparenz ein rosiges Bild. Dennoch bleibt die Frage offen, weshalb die Regierungsführung in der Informationsgesellschaft Eigenschaften aufweisen sollte, die in anderen Bereichen eine Illusion geblieben sind. Weshalb sollten Regierungen, welche die Verletzung der zivilen und politischen Rechte von Menschen ohne Zögern mit der „Terrorismusbekämpfung“ gerechtfertigt haben, das Vertrauen der Bevölkerung in Bezug auf die Errichtung einer Regierungsführung erhalten, bei der die Bürgerbeteiligung im Vordergrund steht? Weshalb sollten grosse, global tätige Unternehmen plötzlich mehr Transparenz gegenüber der Öffentlichkeit und eine verstärkte Ergebnisverantwortung zeigen?

Wie kann die globale Informationsgesellschaft einschliessend und demokratisch sein, wenn die Regierungen nicht bereit sind, auch den WSIS-Prozess demokratisch und einschliessend zu gestalten? Die Bürger haben nicht einmal die Möglichkeit, als gleichberechtigte Partner an einer Konferenz über die Informationsgesellschaft teilzunehmen, denn die Regierungen der UNO-Mitgliedsländer haben dafür gesorgt, dass sie selbst die Kontrolle über den Weltgipfel ausüben, und dass die übrigen Anspruchsgruppen in die abschliessenden Entscheidungen des WSIS nicht eingebunden werden.

\section{„Digitale Kluft" und Entwicklungsunterschiede}

Die Bemühungen zur Überwindung der digitalen Kluft sind ein hervorragendes Beispiel für eine Vision, die jeder gründlichen Analyse entbehrt. Tendenziell gilt dieses Phänomen heute als Folge der weltweiten Verzerrungen in der Verteilung von Informations- und Kommunikationsressourcen. Damit wird das Phänomen der digitalen Kluft von der umfassenden Problematik der Entwicklungsunterschiede isoliert. Tatsächlich jedoch ist der digitale Graben lediglich eines von vielen Erscheinungsbildern der ungleichen Verteilung von materiellen und immateriellen Gütern auf der Welt, und zwar zwischen, wie auch innerhalb der einzelnen Gesellschaften.

Solange dieses zentrale Problem nicht gelöst ist - und dazu wäre ein politischer Wille erforderlich, der in der internationalen Staatengemeinschaft bislang nicht erkennbar ist -, sind feierliche Diskurse über den digitalen Graben lediglich als opportune Ablenkungsmanöver zu bewerten. Letzten Endes stellt die digitale Kluft ein moralisches Dilemma dar, das Richard Rorty folgendermassen umschrieben hat: Keine vorhersehbare technologische Anwendung ist in der Lage, jede Familie so reich zu machen, dass sie ihren Kindern ähnliche Chancen bieten kann wie diejenigen, welche den in den glücklichen Teilen der Welt als selbstverständlich gelten. Ebenso wenig steht zu erwarten, dass die Menschen in den industrialisierten demokratischen Ländern ihren Reichtum so umverteilen, dass die dadurch entstehenden Chancen für Kinder aus unterentwickelten Ländern die Vorteile ihrer eigenen Kinder zunichte machen könnten (1996). Rorty gelangt zum Schluss, dass die Reichen sich nur in einem Szenario als Mitglieder derselben moralischen Gemeinschaft wie die Armen verstehen 
können wenn die Chancen für die Kinder der Armen diejenigen ihrer eigenen Kinder nicht beeinträchtigen (ibid., 1996).

In anderen Worten: Moralische Grundsätze wie menschliche Solidarität werden von den Reichen nur so weit befolgt, als sie ihre eigenen Interessen nicht beschneiden. Stehen die Chancen ihrer eigenen Nachkommen auf dem Spiel, kündigen sie diese Prinzipien auf. Wir alle, die wir in den reichen Regionen der Welt leben, werden mit diesem moralischen Dilemma konfrontiert.

Die von den „Glücklichen“ auf dieser Welt gezeichneten Visionen der Informationsgesellschaft gründen weitgehend auf hehren Zielsetzungen wie der Beseitigung der Armut oder der Verringerung der Einkommensunterschiede. Obgleich einiges davon nicht mehr als offizielle Entwicklungsrhetorik sein mag, darf nicht übersehen werden, dass vieles davon die Überzeugung engagierter Politiker und Akteure vor Ort reflektiert. Wie dem auch sei: Ohne eine erhebliche Einschränkung der Perspektiven der Reichen lässt sich das Los der Armen nicht verbessern.

Damit stehen wir in der Tat vor einer folgenschweren Entscheidung, denn angesichts der begrenzten Ressourcen auf unserem Planeten geht eine Verbesserung der Chancen für die Armen unweigerlich auf die Kosten der Reichen. Eine Erhöhung des Lebensstandards der grossen Mehrheit der „Unglücklichen“ auf das Niveau der privilegierten Minderheit würde zu einem noch nie da gewesenen ökologischen Desaster führen. Sämtliche Entwicklungsbemühungen stehen damit vor der schwierigen Herausforderung, dass sich die Not der Armen nur durch eine Beschneidung der Privilegien der Reichen lindern lässt.

Um diese Herausforderung zu bewältigen, müssten Institutionen und Personen, die ernsthaft an einer Überwindung der Entwicklungsunterschiede interessiert sind, der vorherrschenden neoliberalen Wirtschaftsordnung abschwören, die zu einer Verarmung der Mehrheit der Weltbevölkerung führt und gleichzeitig einer begüterten Minderheit einen noch nie da gewesenen Wohlstand beschert. Dass heute die Einkommensunterschiede überall auf der Welt rapide zunehmen, lässt sich empirisch problemlos nachweisen. 1960 waren die reichsten $20 \%$ der Weltbevölkerung dreissig Mal reicher als die ärmsten 20\%. 1991 betrug dieses Verhältnis 61:1, und 1994 gar 78:1. Der Anteil der ärmsten 20\% am gesamten weltweiten Einkommen betrug 1997 1,1\% (gegenüber 2,3\% im Jahr 1960). 1997 hatten 1,3 Milliarden Menschen von insgesamt 5,7 Milliarden weniger als zwei Dollar pro Tag zur Verfügung, während die 358 reichsten Menschen 45\% des weltweiten Einkommens unter sich aufteilten.

Die Armut, ein Wesenszug der wirtschaftlichen Globalisierung, beschränkt sich längst nicht mehr ausschliesslich auf die armen Länder der südlichen Hemisphäre: In Osteuropa beispielsweise, genauer gesagt in den früheren Teilrepubliken der Sowjetunion, erhöhte sich die Zahl der Armen (die mit weniger als vier Dollar pro Tag auskommen müssen) von 14 Millionen im Jahr 1988 auf 119 Millionen im Jahr 1994. In Westeuropa droht mehr als 70 Millionen Armen der soziale Ausschluss. 
Dagegen lässt sich einwenden, dass zahlreiche Länder nur ein geringes Wirtschaftswachstum aufweisen. In der Tat ist unbestritten, dass ohne wirtschaftliches Wachstum keine Aussicht besteht, die Armut zu beseitigen. Damit jedoch das Wirtschaftswachstum zur Verringerung der Armut beitragen kann, sind gut durchdachte politische Programme zur Entlastung der Armen und Umverteilungspolitiken erforderlich. Ferner zeigt die Erfahrung, dass die durch Wirtschaftswachstum bewirkte Armutsverringerung nicht zwangsläufig von einer Verringerung der sozialen Ungerechtigkeit begleitet wird, denn in den meisten Ländern profitieren die Reichen stärker vom BIP-Wachstum als die Armen. So hat sich beispielsweise in den meisten lateinamerikanischen Ländern das Einkommen der ärmsten $20 \%$ der Bevölkerung verringert, und einiges spricht dafür, dass der so genannte Globalisierungsprozess die ungleiche Verteilung der wirtschaftlichen Vorteile noch verstärkt. Der Welthandelsanteil der am wenigsten entwickelten Länder (die rund $10 \%$ der Weltbevölkerung stellen), der in den 70er-Jahren noch $0,6 \%$ betrug, schrumpfte innerhalb von 20 Jahren auf 0,3\%.

Die aktuellen Debatten um die digitale Kluft beruhen offensichtlich auf der unbegründeten Annahme, dass die Inhaber der IKT ihre Haltung und ihre Politik in Bezug auf den internationalen Technologietransfer revidieren werden. In den vergangenen Jahrzehnten haben die vorherrschenden internationalen Politiken auf dem Gebiet des Technologietransfers schier unüberwindbare Barrieren für die Verringerung des technologischen Grabens zwischen Nord und Süd errichtet.

Anzeichen für einen grundlegenden Wandel bei den Praktiken des Technologietransfers sind nicht in Sicht. Damit dürften sich die Beziehungen zwischen „IKT-reichen“ und „IKT-armen“ Ländern in naher Zukunft kaum verbessern. Ferner bedarf es bedeutender Anstrengungen seitens der internationalen Staatengemeinschaft, um die gemeinsame Nutzung der Kommunikationsinfrastrukturen (von Telekommunikationsanbietern errichtete Übertragungssysteme wie Satelliten, Kabel, Fest- und Mobilnetze), der Rechenleistungen (Computer, Peripheriegeräte, Netzwerke), der Informationsquellen (Datenbanken, Bibliotheken) und der IKT-Kenntnisse (intellektuelle und soziale Fähigkeiten, die für eine gewinnbringende Nutzung der IKT erforderlich sind) gerechter zu gestalten. Für die Erneuerung, Optimierung und Erweiterung von Netzwerken in den Entwicklungsländern, aber auch für Programme zum Wissenstransfer und zur Weitergabe von IKT-spezifischen Fähigkeiten - vor allem auch an Frauen - sind enorme Investitionen erforderlich.

1986 schätzte die Maitland-Kommission den jährlichen Investitionsbedarf auf rund 12 Milliarden USD, um zu Beginn des 21. Jahrhunderts allen Menschen auf der Welt Zugang zum Telefon zu verschaffen (Maitland, 1986). In seiner Ansprache vor der WTO-Ministerkonferenz am 8. Dezember 1996 erklärte der damalige Weltbank-Direktor Gautam S. Kaji, dass die Investitionen in die Telekommunikationsinfrastruktur der Entwicklungsländer, die zwischen 1990 und 1994 jährlich rund 30 Milliarden USD betrugen, für die nächsten fünf Jahre verdoppelt werden müssten, um die erforderliche Erschliessung zu ermöglichen. Da die benötigten Investitionen unmöglich allein aus Steuereinnahmen und aus Geldern der öffentlichen Hand finanziert werden könnten, müsse auch der Privatsektor einen Beitrag leisten (I-Ways Magazine, 1996: 32-34). 
Ob die Erwartung gerechtfertigt ist, dass durch private Finanzierungen die weltweite Gerechtigkeit in Bezug auf Zugang und Nutzung der IKT gefördert wird, sei dahingestellt. Hingegen sollten die Vertreter der internationalen Politik und die Regierungen wohlhabender Länder daran erinnert werden, dass nicht nur unzureichende Mittel, sondern vor allem auch mangelnder politischer Wille Lösungen verhindern. In einer Weltwirtschaft, die jährlich Einkommen von rund 22 Billionen USD erwirtschaftet, sollte die Finanzierung des weltweiten adäquaten Zugangs zu IKT kein Problem sein. Das eigentliche Problem liegt darin, dass die Ausgaben für die Entwicklungszusammenarbeit gerade 0,25\% - nämlich 55 Milliarden USD - dieses Einkommens betragen. Das Entwicklungsprogramm der Vereinten Nationen (UNDP) bestätigte 1998, dass die öffentliche Entwicklungshilfe einen Tiefsstand seit der statistischen Erfassung erreicht habe (UNDP, 1998: 37).

Um die Mittel, die für die Gewährleistung des universellen Zugangs zu grundlegenden IKT-Ausrüstungen und -Dienstleistungen erforderlich sind, einigermassen abschätzen zu können, müssen zusätzlich zu den anfänglichen Investitionen in die Infrastruktur und deren regelmässigen Wartung auch eine Milliarde Telefonanschlüsse, Subventionen für über 600 Millionen Haushalte, die sich die Grundgebühren nicht leisten können sowie die Bereitstellung von Computern und Internetanschlüssen für Schulen berücksichtigt werden. Damit ergeben sich für alle Entwicklungsländer insgesamt jährliche Kosten von 80-100 Milliarden USD über einen Zeitraum von 10 Jahren. Diese Mittel bereitzustellen, sollte eigentlich nicht besonders schwierig sein. Zum Vergleich: Diese Summe entspricht $11 \%$ der jährlichen weltweiten Ausgaben für militärische Zwecke, rund $22 \%$ der gesamten jährlichen Ausgaben für Betäubungsmittel oder den jährlichen Ausgaben Europas für alkoholische Getränke (UNDP, 1998).

Aus verschiedenen politischen und wirtschaftlichen Gründen reduzieren zahlreiche Geberländer derzeit ihre Ausgaben für die IKT-Entwicklung. Zwischen 1990 und 1995 verringerten sich die multilateralen Kredite für Telekommunikation von 1253 auf 967 Millionen USD, während bei der bilateralen Hilfe für Telekommunikationseinrichtungen zwischen 1990 und 1995 ein Rückgang von 1259 auf 800 Millionen USD verzeichnet wurde.

\section{Zentrale Bedeutung der Regulierung}

Bevor die internationale Gemeinschaft der Weltöffentlichkeit verspricht, die bestehende Ungleichheit bei der Verfügbarkeit, beim Zugang und bei der Finanzierbarkeit von Information, Kommunikationsinfrastrukturen und Inhalten (Nachrichten, allgemeine Informationen, wissenschaftliche Erkenntnisse, Kunst) zu beseitigen, muss sie die heute geltenden regulatorischen Grundlagen über Infrastruktur und Inhalte überprüfen. Dazu gehört zumindest die Überarbeitung und Ergänzung von Übereinkommen (beispielsweise der Internationale Fernmeldevertrag), das System für die internationale Verteilung von Einnahmen aus der grenzüberschreitenden Telekommunikation und das Abkommen über handelsbezogene Aspekte der Rechte an geistigem Eigentum (TRIPS-Abkommen). 


\section{Welthandelsorganisation (WTO)}

Die heute geltenden internationalen Übereinkommen auf dem Gebiet der Telekommunikation gründen im Wesentlichen auf der Annahme, dass die Telekommunikationsinfrastruktur eines Landes durch private Unternehmen bewirtschaftet werden kann, und dass der Staat verlustbringende Teile des Netzes mit öffentlichen Geldern subventioniert und dafür sorgt, dass kein Bürger auf seinen Anschluss verzichten muss. Anlässlich des zweiten Telecommunication Policy Forum on Trade in Telecommunication Services (Genf, 16. bis 18. März 1998) umriss der damalige ITU-Generalsekretär Pekka Tarjanne die Grundzüge des globalen Regimes im Telekommunikationsbereich. Tarjanne wies darauf hin, dass sich dieser Sektor mit der Einführung des WTO-Abkommens über BasisTelekommunikationsdienstleistungen und demjenigen über den Handel mit Dienstleistungen (GATS-Abkommen) grundlegend verändert habe.

Der Telekommunikationssektor untersteht heute einem handelsrechtlichen Rahmen. Im Übergang zu einem marktorientierten Umfeld müssen alle Länder die Gelegenheit erhalten, sämtliche Chancen zu nutzen, die ein liberalisierter und dynamischer weltweiter Telekommunikationsmarkt bietet.

Diese Grundsätze sind im internationalen Vertrag über Telekommunikationsdienstleistungen verankert. Mit dem Abschluss des Abkommens zur Errichtung der Welthandelsorganisation am 15. April 1994 in Marrakesch wurde die achte multinationale GATT-Verhandlungsrunde (Uruguay-Runde) offiziell beendet. Das Schlussabkommen umfasste auch ein Allgemeines Abkommen über den Handel mit Dienstleistungen (GATS-Abkommen), dessen wohl komplexester Anhang den Handel mit Telekommunikationsdienstleistungen betrifft. In diesem Anhang werden Basis-Telekommunikationsdienstleistungen und -netzwerke folgendermassen definiert:

๖ öffentliche Telekommunikationsdienstleistung: jede Art von Telekommunikationsdienstleistung, die einer ausdrücklichen oder tatsächlichen Forderung des Mitglieds gemäss der breiten Öffentlichkeit anzubieten ist.

$\checkmark$ öffentliches Telekommunikationsnetz: die öffentliche Infrastruktur, welche die Telekommunikation zwischen zwei oder mehr definierten Netzabschlüssen ermöglicht.

Rund 60 der 125 Unterzeichnerstaaten des Abkommens von Marrakesch verpflichteten sich, ihren Markt für Telekommunikationsdienstleistungen zu öffnen, wobei die meisten von ihnen in Bezug auf Basis-Telekommunikationsdienstleistungen keine Verpflichtungen eingingen. Die Verpflichtungen reichen von uneingeschränktem Wettbewerb für alle Telekommunikationsdienstleistungen zu Ausnahmen für Basis-Telekommunikationsdienstleistungen, Mobilfunkoder lokale Dienstleistungen. Ferner wurde in Marrakesch die Verhandlungsgruppe für Basis-Telekommunikationsdienstleistungen eingesetzt, die bis April 1996 eine abschliessende Lösung für den Handel mit Telekommunikationsdienstleistungen erarbeiten sollte.

Bis heute ist die Verhandlungsgruppe zu keiner Einigung gekommen. In mehreren Punkten, etwa in der Liberalisierung der Satellitennutzung und in Bezug auf 
die Streitbeilegung bei internationalen Telekommunikationsgebühren, bestehen noch Divergenzen. Immerhin führten die Verhandlungen zur Vereinbarung einiger grundlegender Regeln, die in einem so genannten Referenzpapier festgehalten sind. Das Referenzpapier regelt Punkte wie Wettbewerbsschranken, Interkonnektivität, Verpflichtung zum Universaldienst, Transparenz bei der Lizenzvergabe, Unabhängigkeit der regulierenden Behörde sowie den Einsatz knapper Ressourcen.

Ab Juli 1996 wurden die Arbeiten von der Gruppe für Basis-Telekommunikationsdienstleistungen weitergeführt. Die Gruppe stand allen WTO-Mitgliedsländern offen und tagte monatlich. Ihre Aufgabe bestand darin, zusätzliche Länder $\mathrm{zu}$ Verpflichtungen zu bewegen, sich mit der Liberalisierung von Satellitendienstleistungen zu befassen und in bestimmten Fragen in Bezug auf die Bereitstellung von Telekommunikationsdienstleistungen eine Lösung herbeizuführen.

Diese neue Verhandlungsrunde befasste sich unter anderem mit den Beschränkungen ausländischer Beteiligungen. Insbesondere die USA drängten auf grösstmögliche ausländische Beteiligungen auf den inländischen Telekommunikationsmärkten. In ihren Verpflichtungen verzichteten zahlreiche Länder vollständig auf eine Beschränkung ausländischer Beteiligungen, während andere eine inländische Kontrolle des Marktes zwischen 25\% und 80\% als Bedingung stellten. Einige Länder betrachten eine ausländische Beteiligung als Chance, ausländische Investitionen anzuziehen (ITU, 1997: 102), andere wiederum sehen darin eine Gefahr für die nationale Souveränität. Die nationalen Regierungen können den Umfang, die zeitliche Entwicklung und das In-Kraft-Treten ihrer Verpflichtungen frei bestimmen. Allerdings können diese Verpflichtungen im nachhinein nicht neu verhandelt werden.

Das vierte Protokoll des Allgemeinen Abkommens über den Handel mit Dienstleistungen wurde am 15. Februar 1997 von 72 WTO-Mitgliedsländern (die gemeinsam rund $93 \%$ des weltweiten Handels mit Telekommunikationsdienstleistungen abdecken) unterzeichnet und trat am 5. Februar 1998 in Kraft. Diese weltweit geltende Übereinkunft über Telekommunikationsdienstleistungen verpflichtet die Mitglieder zur Liberalisierung ihrer Märkte. Obwohl einige Bestimmungen es den Ländern ermöglichen, die Umsetzung des universalen Zugangs nach eigenen Bedürfnissen zu gestalten, enthält die Vereinbarung Vorgaben von bedeutender Tragweite, die den nationalen politischen Spielraum massgeblich einschränken.

Die Vereinbarung hat weit reichende Konsequenzen für die Organisation der grundlegenden Telekommunikations-Infrastrukturen. In Bezug auf den Universaldienst hält das Abkommen fest, dass jedes Mitglied berechtigt ist, Art und Umfang des Universaldienstes, den es aufrecht erhalten will, zu definieren. Solche Verpflichtungen gelten nicht als per se wettbewerbswidrig, solange sie transparent, nicht-diskriminierend und wettbewerbsneutral umgesetzt werden und nicht über das Mass hinausgehen, das für den Schutz des vom jeweiligen Land definierten Universaldienstes erforderlich ist. Damit haben sich die Signatarstaaten dazu verpflichtet, die lokalen Bedingungen für den universalen Zugang nicht stärker als notwendig (more burdensome than necessary) $\mathrm{zu}$ 
erschweren, wodurch der Spielraum für eine unabhängige nationale Politik in Bezug auf den Zugang stark eingeschränkt wird. Da ausländische Anbieter gegenüber inländischen nicht schlechter gestellt werden dürfen, müssen die Standards des Universaldienstes wettbewerbsneutral umgesetzt werden. Namentlich dürfen sie das ,notwendige“ Mass nicht überschreiten. Sollte eine nationale Politik darauf abzielen, den Zugang zu Telekommunikationsdienstleistungen über Quersubventionierungen, anstatt auf der Grundlage kostengestützter Tarife sicherzustellen, käme dies den Interessen kleiner Nutzer mehr entgegen als denen der Betreibergesellschaften. Aus der Sicht ausländischer Anbieter hingegen dürfte eine solche Verpflichtung das „notwendige“ Mass übersteigen, und die entsprechende Politik könnte als Verstoss gegen das internationale Handelsrecht gewertet werden. Damit läge es an dem (weitgehend obskuren) Streitbeilegungsmechanismus der WTO, über die Rechtmässigkeit bzw. Rechtswidrigkeit der nationalen Politik zu befinden.

Das Telekommunikationsabkommen misst dem Zugang ausländischer Anbieter zu den nationalen Telekommunikationsmärkten grösseres Gewicht bei als dem Zugang der Bürger zur Nutzung der angebotenen Telekommunikationsdienstleistungen. Es wäre vereinfachend $\mathrm{zu}$ behaupten, diese beiden Formen des Zugangs würden sich decken.

Als Folge stossen gesellschaftsrelevante politische Bemühungen an Grenzen, die von den Akteuren der Wirtschaft gezogen werden. Die nationalen Politiken im Telekommunikationsbereich werden stärker durch Handelsinteressen als durch soziokulturelle Anliegen geprägt. Auf der Grundlage des Abkommens und der Tatsache, dass die Mehrzahl der Handelspartner sich zur Liberalisierung ihrer nationalen Märkte verpflichtet haben, geht die WTO davon aus, dass bis 2004 ein nahezu weltweiter (bis zu 93\%) offener Markt für Basis-Telekommunikationsdienstleistungen entstanden sein wird. Allerdings bedeutet die Schaffung weltweiter freier Märkte für jede Art von Dienstleistungen nicht zwangsläufig, dass solche Dienstleistungen überall verfügbar sind, oder dass sie von all denen, die davon profitieren könnten, auf einer gerechten Basis genutzt werden können.

Die wachsende Beteiligung in den Verhandlungen über den Telekommunikationssektor und die zunehmenden Verpflichtungen zur Marktöffnung legen den Schluss nahe, dass immer mehr Länder davon ausgehen, eine Liberalisierung ihres Telekommunikationsmarktes sei für sie von Vorteil. Die politische Frage lautet nicht so sehr, ob liberalisiert werden soll oder nicht, sondern wann. Nach wie vor aber gehen die Meinungen auseinander, wie beispielsweise der World Telecommunication Report festhält: Der Marktzugang beispielsweise wird von einigen Akteuren als Chance wahrgenommen, während andere, die den Aufbau einer eigenen nationalen Telekommunikationsindustrie fördern wollen, darin eine Gefahr und eine Bedrohung für im Entstehen begriffene nationale Anbieter sehen (ITU, 1997: 102).

Während die Liberalisierung in einigen Ländern zusätzliche Einnahmen für inländische Anbieter generieren wird, dürfte in anderen Staaten der Grossteil der Erträge ausländischen Unternehmen zufliessen. Der ITU-Bericht weist zu Recht darauf hin, dass es Gewinner und Verlierer geben wird (1997: 106). 


\section{Wachsende Zahl von Privatisierungen}

Im Zuge der Öffnung ihrer Märkte haben zahlreiche Länder begonnen, ihre öffentlichen Anbieter von Telekommunikationsdienstleistungen (public telecom operators, PTO) zu privatisieren. Seit 1984 gingen 44 PTO an private Eigentümer über (ITU 1997: 2). Diese Privatisierungen setzten rund 159 Milliarden USDollar frei. Allein die 12 grössten Privatisierungsprojekte im Jahr 1996 generierten Mittel von mehr als 20 Milliarden USD. Die Investitionen stammten je rund zur Hälfte aus dem In- und Ausland. Ingesamt wurden über $30 \%$ der Investitionen von ausländischen Kapitalgebern getätigt. Laut dem ITU-Bericht sind die aktivsten Investoren in der Regel unter den PTO selbst zu suchen. Allerdings befanden sich 1997 die Mehrheit der Anteile von 29 der 40 grössten internationalen Anbieter nach wie vor im Staatsbesitz. In sämtlichen Regionen wurden ehemals staatliche Telekommunikationsunternehmen eher in Kapitalgesellschaften umgewandelt als privatisiert (ITU, 1998: 9). Zudem hat die Liberalisierung noch nicht in allen Ländern zu sektorenübergreifendem Wettbewerb geführt. In zahlreichen Ländern sind die Basis-Telekommunikationsdienstleistungen vom Wettbewerb ausgenommen. Die Mobiltelefonie gehört zu den am stärksten liberalisierten Märkten, aber selbst in diesem Sektor wird der Wettbewerb in einigen Ländern (noch) unterbunden.

Als Argumente für die Privatisierung dienen die Erweiterung und die Optimierung von Netzwerken, die Verbesserung des Dienstleistungsangebots und die Tarifsenkungen für den Zugang zu den Netzen und deren Nutzung. Allerdings wurden bisher sehr unterschiedliche Erfahrungen gemacht. In manchen Ländern sind die Tarife in der Tat gesunken - aber hauptsächlich für bedeutende Geschäftskunden, während die Telefonrechnungen der Durchschnittskunden kaum zurückgingen. Auch in Bezug auf das Dienstleistungsangebot bietet sich ein gemischtes Bild. Dies ist zum Teil darauf zurückzuführen, dass die Privatisierungen die Erwartungen in Bezug auf mehr Wettbewerb und mehr Auswahl nicht immer erfüllen konnten. Tatsächlich waren die nationalen Telekommunikationsanbieter in den kleineren und weniger fortgeschrittenen Ländern den grossen globalen Koalitionen - den neuen Monopolisten - unterlegen. Es ist zu bezweifeln, ob von wenigen globalen Anbietern beherrschte Märkte dem Konsumenten einen Nutzen bringen werden. Fraglich ist auch, wie viel Wettbewerb letztlich vorhanden sein wird, denn Preissenkungen und Investitionen in technologische Innovationen bewirken, dass Konkurrenzunternehmen aus dem Markt gedrängt werden und die Liberalisierung der Märkte fast überall zu einer Konzentration auf der Anbieterseite führt.

Am meisten Rätsel gibt jedoch die allgemeine Erwartung auf, wonach die Marktliberalisierung einen von Wettbewerb geprägten Markt hervorbringen wird. Die Geschichte zeigt, dass freie Märkte unweigerlich die Bildung von Monopolen begünstigen, da konkurrierende Unternehmen einander entweder aus dem Markt drängen oder aber miteinander fusionieren. Die Kernfrage lautet somit nicht, ob Monopole oder freier Wettbewerb zu bevorzugen sind, sondern ob staatliche Monopole weniger effizient und effektiv sind als privatwirtschaftliche Monopole. 
Nicht selten führen Privatisierungen zu einer Ausdehnung der Telekommunikationsnetze. In einigen Länder (z. B. in Peru 1997 und in Panama 1997) hat die Anschlussdichte im Zuge der Privatisierung markant zugenommen. Einer der Gründe dafür ist gemäss ITU die Tatsache, dass der Ausbau der Netze immer häufiger als Bedingung für die Vergabe von Privatisierungskonzessionen gestellt wurde (1998: 71). Selbstverständlich bringen die zusätzlichen Telefonanschlüsse jenen Nutzern Vorteile, die es sich leisten können. Der Kreis der Bürger mit der für die Nutzung von Telekommunikationsnetzen erforderlichen Kaufkraft wird jedoch durch die Privatisierung nicht erweitert.

Der lukrative Tätigkeitsbereich eines nationalen Telekommunikationsanbieters sind die Auslandsverbindungen. Allerdings fliessen die finanziellen Gewinne aus den Überseeverbindungen nicht zwingend dem nationalen Anbieter zu. Aus politischen und anderweitigen Gründen sehen zahlreiche Länder davon ab, einen ausreichenden Teil dieser Erträge in die Erweiterung und den Ausbau der Telekommunikationseinrichtungen zu investieren. Zudem intensiviert sich der Wettbewerb im Bereich der Auslandverbindungen laufend, und auf den wichtigen Linien herrscht ein starker Preisdruck.

\section{Verteilung der Erträge}

Die Verfügbarkeit von Finanzmitteln für den Auf- und Ausbau der Telekommunikationsinfrastrukturen hängt auch von den internationalen Vereinbarungen betreffend die Aufteilung der Erträge aus der grenzüberschreitenden Telefonie ab. Eines der wichtigsten Ziele der Regulierung der Telekommunikation, das im ersten Internationalen Fernmeldevertrag (ITU, 1985) verankert wurde, war die Errichtung eines adäquaten Systems für die Aufteilung der Erträge aus der internationalen Telefonie zwischen Herkunfts-, Transit- und Bestimmungsländern. Dieses so genannte Telefongebühren-Aufteilungssystem sieht vor, dass der PTO im Herkunftsland dem Kunden einen bestimmten Tarif in Rechnung stellt, und der PTO des Bestimmungslandes mit jenem des Herkunftslandes anschliessend einen Preis für die Leistungen vereinbart, die der PTO im Bestimmungsland erbringt (Bereitstellung internationaler Linien, der Verbindung und der Weiterleitung des Gesprächs an den lokalen Kunden). Dieser Betrag bildet die Grundlage für die so genannten settlement rates, welche die Telefonbetreiber im Bestimmungsland dem Betreiber im Herkunftsland in Rechnung stellen. Im Allgemeinen hat die ITU empfohlen, die Gebühren je zur Hälfte auf die Betreiber aufzuteilen, eine Lösung, die in einem von Monopolen geprägten Umfeld, in dem die internationale Telefonie als gemeinsam erbrachte Dienstleistung galt, tadellos funktionierte. Dies änderte sich jedoch mit der wachsenden Anzahl privater Anbieter, dem zunehmenden Wettbewerb und den technischen Möglichkeiten, das bestehende System zu umgehen.

Seit einiger Zeit beschäftigen sich die Organisation für wirtschaftliche Zusammenarbeit und Entwicklung (OECD, seit 1991), die ITU (seit 1992) und die WTO mit einer Reform des bestehenden Systems zur Gebührenaufteilung. In der Vergangenheit kam das bisherige System den Interessen der Entwicklungsländer entgegen, denn ihre Gebühren für die Weiterleitung internationaler Gespräche an Kunden in ihrem Land waren in der Regel relativ hoch, so dass 
die Aufteilung der Gebühren eine bedeutende Einnahmequelle von Devisen darstellte. Zumindest im Prinzip können diese Einnahmen dazu verwendet werden, den Zugang zu Telekommunikationsinfrastrukturen in ländlichen Gegenden zu verbessern.

Laut ITU fliessen jährlich 10 Milliarden US-Dollar als Nettozahlungen in die Entwicklungsländer. Als die Verhandlungen nicht im gewünschten Tempo vorankamen, entschieden die US-amerikanischen Behörden, ihre bevorzugte Lösung bekannt zu geben. Die US Federal Communications Commission argumentierte, die USA verlören durch die Zahlungen an andere Länder jedes Jahr mehrere Millionen Dollar, und führte im November 1996 ein revidiertes System ein (die so genannte Notice of Proposed Rulemaking, die im Januar 1998 in Kraft trat), welches festlegt, welchen Betrag US-amerikanische Betreiber an ausländische Anbieter bezahlen müssen (im Durchschnitt die Hälfte des bisherigen Betrags).

Im Rahmen ihrer Liberalisierungs- und Wettbewerbspolitik entschied die Europäische Union, die Gebührenaufteilung für Gespräche zwischen den EU-Mitgliedsländern ausser Kraft zu setzen und stattdessen ein kostenbasiertes System für die Verrechnung von Dienstleistungen für die Netzzusammenschaltung (so genannte interconnection services) einzuführen.

Kritiker des bisherigen Systems machten geltend, dass über den tatsächlichen Kosten liegende Zahlungen in einem zunehmend von wirtschaftlichem Denken geprägten Telekommunikationsmarkt nicht mehr angebracht seien. Es sei inakzeptabel, dass das anrufende Land überhöhte Preise für die Herstellung von Verbindungen bezahlen müsse, damit das Bestimmungsland seine Politik zur Gewährleistung des universalen Zugangs subventionieren könne. Die Verfechter des bestehenden Systems verweisen auf die grosse Bedeutung dieser Zahlungen für die Gewährleistung des universalen Zugangs in armen Ländern und argumentieren, eine Verringerung der Zahlungen würde die Kosten für die Kunden in diesen Ländern erhöhen. Die Ausgestaltung eines etwaigen neuen Systems zur Gebührenaufteilung wird somit nicht nur für den Zugang zu IKT in ärmeren Ländern, sondern auch für die allgemeine wirtschaftliche Lage dieser Länder ausschlaggebend sein.

\section{Rechte am geistigen Eigentum}

Ein weiterer wichtiger Aspekt der internationalen Regulierungstätigkeit ist der Schutz der geistigen Eigentumsrechte (intellectual property rights, IPR). Von Beginn an beruhte der Schutz des geistigen Eigentums auf drei Motiven. Erstens wurde davon ausgegangen, dass diejenigen, die in die Produktion von geistigem Eigentum investiert haben, eine finanzielle Entschädigung erhalten sollen. Seit dem Abschluss der ersten internationalen Verträge über den Schutz des geistigen Eigentums (der Pariser Verbandsübereinkunft zum Schutze des gewerblichen Eigentums von 1883 und der Berner Übereinkunft zum Schutze von Werken der Literatur und Kunst aus dem Jahr 1886) gilt ein finanzieller Nutzen für den Urheber als notwendiger Anreiz für Investitionen in Innovation und Kreativität. Im Zuge der Revision der Berner Übereinkunft von 1982 wurde zusätzlich 
zum Recht auf wirtschaftlichen Nutzen das Konzept der moralischen Rechte verankert. Damit wurde anerkannt, dass die Werke Ausdruck der intellektuellen Persönlichkeit des Urhebers sind. Die moralischen Rechte schützen das schöpferische Werk vor Veränderungen ohne Zustimmung des Urhebers und gewährleisten den Anspruch auf Urheberschaft und das Recht des Urhebers, über die Veröffentlichung eines Werks zu entscheiden.

Bereits früh in der Entwicklung der geistigen Eigentumsrechte wurde anerkannt, dass der Schutz des geistigen Eigentums im Interesse der Öffentlichkeit liegt. Nach allgemeiner Auffassung fördern die IPR die Innovation und den Fortschritt in Kunst, Technologie und Wissenschaft, und damit auch die Wohlfahrt der Allgemeinheit. Somit gestaltet sich der Schutz des geistigen Eigentums als Balanceakt zwischen Interessen der Privatwirtschaft, individuellem Eigentum, moralischen Werten und dem öffentlichen Interesse. In den vergangenen Jahren hat sich das internationale Regelwerk von den moralischen Werten und dem Interesse der Öffentlichkeit distanziert und in der Praxis die wirtschaftlichen Interessen der Eigentümer von geistigen Eigentumsrechten in den Vordergrund gerückt. Diese Eigentümer sind heute längst nicht mehr einzelne Autoren und Komponisten, die Güter von kulturellem Wert schaffen, sondern international tätige, Kultur produzierende Unternehmen. Für sie ist der Kampf gegen die „Piraterie“ und die dadurch entstehenden Verluste wichtiger als der Schutz der moralischen Integrität kreativer Werke oder die Qualität des kulturellen Lebens auf der Welt.

Das Abkommen über handelsbezogene Aspekte der Rechte am geistigen Eigentum (TRIPS-Abkommen), das 1993 im Zuge der Uruguay-Runde der multilateralen Handelsverhandlungen als Anhang 1C des Allgemeinen Zoll- und Handelsabkommens entstanden ist, wird heute im Rahmen der WTO-Regelungen umgesetzt. Mit dem TRIPS-Abkommen wurde die ökonomische Dimension des Schutzes des geistigen Eigentums verstärkt. Wie Shalini Venturelli zu Recht feststellt, hat sich das Gleichgewicht radikal verlagert, und zwar zu Gunsten wirtschaftlicher Interessen und Anreizen für dritte Nutzer, und zum Nachteil des öffentlichen Zugangs der Bürger und verfassungsrechtlicher sowie individueller Rechte an kreativer Arbeit (Venturelli 1998: 63). Heute sind die Rechte des geistigen Eigentums eines der wichtigsten handelbaren Güter auf der Welt, und das gegenwärtige Regelwerk begünstigt die produzierenden Unternehmen (Verleger, Rundfunkanstalten, Musikproduzenten, Werbeagenturen) gegenüber den einzelnen Urhebern. Die wirtschaftlichen Ansprüche der Investoren sind durch das TRIPS-Abkommen besser geschützt als die moralischen Rechte kreativer Personen oder die kulturellen Interessen der breiten Öffentlichkeit.

Deutlich erkennbar wurde dieser Trend zum verstärkten Schutz der wirtschaftlichen Interessen produzierender Gesellschaften Ende 1996, als die US-Regierung und die Europäische Union Vorstösse einbrachten, wonach digitale Datenbanken und digitale Kopien (auch vorübergehender Natur) ebenfalls durch die Berner Übereinkunft geschützt werden sollten. Zur Unterstützung dieser Vorstösse bildete sich eine mächtige Lobby, welche die Interessen der Motion Picture Association, der International Federation of Phonographic Industries, der Federation of European Publishers sowie der Business Software Alliance - der 
Unternehmen wie Microsoft, Apple und IBM angehören - vertrat und von ihnen finanziell unterstützt wurde. Sie schlugen vor, Artikel 7 der revidierten Übereinkunft durch eine Bestimmung zu erweitern, wonach die direkte und indirekte Reproduktion in jeder Form, sei sie dauerhaft oder vorübergehender Natur, ebenfalls urheberrechtlichen Schutz erhalten sollte. In Bezug auf die Nutzung von Internet-Quellen würde dies die Errichtung eines „Pay per View“-Systems voraussetzen. Die Gegner dieses Vorschlags (eine Lobby, in der Telekommunikationsanbieter wie AT\&T, Philips, British Telecom und France Telecom sowie Netscape, Bibliotheken, Internetanbieter und Internetnutzer vertreten waren) hielten dagegen, Artikel 7 der Übereinkunft sei der Attraktivität des Internets abträglich und führe zu einer wesentlichen Beeinträchtigung des freien Zugangs zu Information.

Am 20. Dezember 1997 entschied die Konferenz die Streichung des umstrittenen Artikels 7. Damit war - zumindest vorläufig - dem Ausbau des Urheberrechtsschutzes Einhalt geboten. Allerdings ist fraglich, ob sich die Befürworter mit dieser Niederlage geschlagen geben. Die EU-Richtlinie über Datenbanken (11. März 1996) lässt darauf schliessen, dass die Anstrengungen zur Ausweitung des Urheberrechtsschutzes auf digitale Informationsquellen unvermindert fortgesetzt werden. Die Richtlinie unterstellt den freien Zugang zu Datenbanken den Bestimmungen des Urheberrechtsschutzes.

Als Schlüsselargument zu Gunsten einer Stärkung des Schutzes von geistigem Eigentum (namentlich durch eine Ausdehnung der Rechte und durch die strikte Ahndung von Verstössen) dienen in der Regel die Verluste (von mehreren Milliarden Dollar), welche der Unterhaltungs- und Kulturindustrie auf Grund der weit verbreiteten weltweiten Piraterie entstehen. US-amerikanische Softwarefirmen sowie Unternehmen aus der Unterhaltungs- und Verlagsbranche behaupten, sie hätten 1995 rund 14 Milliarden Dollar an die Piraterie verloren. Die International Federation of Phonogram and Videogram Producers schätzt, dass rund $25 \%$ aller Musikaufnahmen Raubkopien sind. Ein zentrales Problem des derzeitigen Trends zum verstärkten Schutz der Immaterialgüterrechte ist die Tatsache, dass das im Entstehen begriffene Regelwerk die Unabhängigkeit und Vielfalt des weltweiten kreativen Schaffens erstickt. Besonders „kleine“ und unabhängige Urheber kreativer Werke werden durch dieses Regime höchst unzureichend geschützt.

Der Schutz des geistigen Eigentums führt zu kaum überwindbaren Hürden für die Nutzung kreativer Erzeugnisse, denn die Gültigkeit des Konzepts der „angemessenen Nutzung" (fair use), das in der Vergangenheit eine ungehinderte Verwendung dieser Erzeugnisse für zahlreiche Zwecke - auch im Bildungsbereich - ermöglichte, wird beschnitten. Aus seinem engen, ökonomisch geprägten Blickwinkel betrachtet, drängt das Motiv der ungerechtfertigten Nutzung von Unternehmenseigentum dasjenige der Innovationskraft künstlerischen und literarischen Schaffens in den Hintergrund. Besonders bedenklich stimmt die Tatsache, dass kulturelle Erzeugnisse aus dem „Öffentlichkeitsbereich“ ebenfalls unter den Schutz der Immaterialgüterrechte fallen, sobald sie in einer elektronischen Datenbank gespeichert werden. Damit wird der freie Zugang zu Informationsquellen in bedeutendem Umfang eingeschränkt. Nicht zuletzt droht das 
neue globale Forum, das der Cyberspace (über die neuen IKT) möglicherweise bieten kann, zu einem Markt zu verkommen, auf dem eine kontrollierte Menge an Ideen kommerzialisiert wird.

\section{UNCTAD-Kodex als Lösung?}

Zusätzlich zu einer minimalen Überarbeitung und Änderung der erwähnten internationalen Vereinbarungen ist die Staatengemeinschaft aufgefordert, den von der UNCTAD vorgeschlagenen internationalen Verhaltenskodex für den Technologietransfer (Code of Conduct on the Transfer of Technology) zu verabschieden. Seit 1997 wurden verschiedene Entwürfe dieses Kodexes erörtert, aber eine Einigung lässt noch immer auf sich warten. Der Kodex zielt im Wesentlichen darauf $a b$, einen fairen Rahmen zu schaffen, der es ärmeren Ländern erlaubt, ebenfalls vom technologischen Fortschritt zu profitieren und sie nach und nach in die Lage zu versetzen, ihre eigenen technologischen Innovationsprozesse zu steuern. Das grösste Hindernis für eine international geltende Vereinbarung über den Technologietransfer ist die Tatsache, dass die mächtige Lobby der Technologieanbieter keinen Vorteil darin erkennen kann, dass die Länder der Dritten Welt technisches Know-how sowie die Möglichkeit erwerben, aggressiven Geschäftspraktiken transnationaler Technologieunternehmen entgegentreten zu können.

\section{Vision und Macht}

Angesichts der gegenwärtigen internationalen politisch-wirtschaftlichen Konstellation ist davon auszugehen, dass der WSIS als Schauplatz für die hinlänglich bekannte Konfrontation zwischen Vision und Macht dienen wird. Nichts in den wohlmeinenden Aussagen im Vorfeld des Gipfels deutet darauf hin, dass dieses Mal die Vision die Oberhand gewinnen wird. Ob es eine realistische Chance gibt, dass die Visionen wahr werden, hängt letzten Endes von den globalen Machtverhältnissen ab. Dabei gilt es zu bedenken, dass derzeit auf der weltpolitischen Bühne die Entscheidungen von einigen wenigen Regierungsorganisationen (wie zum Beispiel die Welthandelsorganisation) und privaten Initiativen (unter anderem der Global Business Dialogue on e-Commerce) getroffen werden.

Im vergangenen Jahrzehnt hat das weltweite kommunikationspolitische Umfeld einige grundlegende Entwicklungen durchlaufen. Die Wichtigsten davon werden im Folgenden genannt:

$\square$ In den vergangenen hundert Jahren diente das internationale Regime im Kommunikationsbereich hauptsächlich der Koordination zwischen nationalen Politiken, die von souveränen Regierungen unabhängig voneinander definiert wurden. Heute wird der Spielraum der nationalen Regierungen für ihre eigene politische Tätigkeit weitgehend vom supranational agierenden globalen Regime abgesteckt.

$\square$ Die weltweite Kommunikationspolitik wird zunehmend durch Standards des Handels und des Marktes geprägt, während politische Erwägungen mehr und mehr in den Hintergrund treten. Der politische Diskurs wird zusehends 
durch einen von Wirtschafts- und Handelsanliegen geprägten Diskurs verdrängt. Ein Beweis dafür ist die wachsende wirtschaftliche Bedeutung des geistigen Eigentums und die damit einhergehende stärkere Gewichtung des Schutzes von Investoren und Produzenten. Im Telekommunikationsbereich wurden die Grundsätze des Universaldienstes und der Quersubventionierungen durch kostenbasierte Tarifstrukturen abgelöst. Auf dem Gebiet des grenzüberschreitenden elektronischen Datenverkehrs schliesslich wurden politische Argumente, welche auf nationaler Souveränität und kultureller Eigenständigkeit beruhen, durch Konzepte wie Handelsschranken und Marktzugang verdrängt.

- Die ausserordentlich mächtigen privaten Akteure treten mit ihren Anliegen mehr und mehr in die Öffentlichkeit. Die „unsichtbare Hand“ der wirtschaftlichen Interessen, welche schon immer die politische Entscheidungsfindung massgeblich beeinflusst, ist in den vergangenen Jahren immer sichtbarer geworden. Multinationale Unternehmen sind heute wichtige Akteure auf der weltpolitischen Bühne und rücken ihre Bedeutung bewusst in den Vordergrund. In der Tat wird die Politik heute nicht mehr von Regierungen, sondern von Interessensverbänden und privatwirtschaftlichen Akteuren geprägt. Ein aktuelles Beispiel dafür ist der Global Business Dialogue, welcher 1998 von Unternehmen auf dem Informationssektor mit dem Ziel gegründet wurde, sich mit Regulierungsfragen im Zusammenhang mit dem Internet und insbesondere mit dem elektronischen Handel zu befassen.

- Traditionell wurde die internationale Kommunikationspolitik von regierungsübergreifenden Gremien wie der UNESCO, der Weltorganisation für geistiges Eigentum und der internationalen Fernmeldeunion beherrscht. Diese Organisationen standen der soziokulturellen Dimension der Veränderungen auf dem Gebiet der Informations- und Kommunikationstechnologien relativ aufgeschlossen gegenüber. Zudem boten sie eine Plattform, welche es auch den Entwicklungsländern ermöglichte, ihre Anliegen einzubringen. In den vergangenen Jahren wurde die Stellung dieser zwischenstaatlichen Organisationen geschwächt, da die bedeutendsten Akteure einem Forum den Vorzug gaben, das ihren jeweiligen Interessen förderlicher war. Dieses Forum ist die Nachfolgeorganisation des Allgemeinen Zoll- und Handelsabkommens: die Welthandelsorganisation. Die Gründung der WTO war eines der Ergebnisse der multilateralen Handelsverhandlungen des GATT, der so genannten Uruguay-Runde, die im Dezember 1993 abgeschlossen wurde. Im Allgemeinen steht die WTO den Handelsinteressen der grössten Industrieländer offener gegenüber als andere zwischenstaatliche Organe. Zentrale politische Grundsätze der WTO sind die weltweite Liberalisierung der Märkte, die Nichtdiskriminierung von konkurrierenden ausländischen Anbietern auf dem inländischen Markt sowie die Meistbegünstigungsklausel.

Angesichts der zunehmenden wirtschaftlichen Bedeutung der Kommunikationsnetzwerke und Informationsdienstleistungen überrascht es nicht, dass sich die Zuständigkeit für die Kommunikationspolitik auf dieses handelspolitische Forum verlagert hat. Heute erwirtschaftet der weltweite Kommunikationsmarkt jährlich rund 3000 Milliarden Dollar. Zudem wird der Grossteil der für den Handel mit Gütern und Finanzdienstleistungen erforderlichen Strukturen von 
den bedeutendsten Unternehmen im Kommunikations- und Informationsbereich bereitgestellt. Dies erklärt, weshalb die Politik in kommunikationsrelevanten Bereichen heute weitgehend vom weltweiten Handelsregime abhängt.

Die weltweite Kommunikationspolitik durchläuft somit einen historischen Wandel vom Universaldienst hin zu privatwirtschaftlichem Wettbewerb und wirtschaftlichem Nutzen. Am Ende dieses Prozesses dürften die Interessen der Öffentlichkeit auf der Verliererseite stehen. Das derzeitige globale Regime im Kommunikations- und Informationsbereich ist primär darauf ausgerichtet, Einmischungen der öffentlichen Hand zu minimieren und das freie Spiel der Marktkräfte zu maximieren. Einen Gegenpol zum dominierenden globalen Regime auf dem Gebiet der Kommunikation mit ihren marktorientierten Visionen der Informationsgesellschaft bilden die auf Bürgerbeteiligung beruhenden Visionen, welche von Personen und Organisationen vertreten werden, die von der Bedeutung ihrer Anliegen überzeugt sind und daran glauben, dass die Innovationen im Bereich der Informations- und Kommunikationstechnologie neue Chancen für deren Umsetzung schaffen. Zum Teil werden solche Visionen aber auch von Personen und Organen geäussert, die damit eine in Tat und Wahrheit nicht existierende Bereitschaft und Offenheit für soziale Veränderungen vortäuschen. Fazit: Die in den vorbereitenden Texten zum WSIS gebotenen Visionen der Informationsgesellschaft gründen entweder auf einer beeindruckenden Naivität oder aber auf einer gehörigen Portion Unehrlichkeit.

Was sollen die Menschen auf dieser Welt von einem weiteren selbstgefälligen und kostspieligen internationalen Anlass halten, der ihre tägliche Not nicht im Geringsten lindert? Das beunruhigende Ausmass des weltweiten Misstrauens in Bezug auf die Fähigkeit politischer Institutionen, die Interessen der Menschen zu wahren, dürfte dadurch noch verschärft werden.

Entgegen aller Verheissungen der WSIS-Dokumente wird der Menschheit in Tat und Wahrheit eine globale Informationsgesellschaft von oben aufgezwungen, die folgende Merkmale aufweist:

$\square$ Die Bewegungen der Bürger werden von Vollzugsbehörden und Geheimdiensten rund um die Uhr überwacht.

$\checkmark$ Die über einzelne Bürger (auch solche, die nicht als Kriminelle verdächtigt werden) gesammelten Daten werden für eine unbestimmte Dauer aufbewahrt.

$\checkmark$ Die Besitzrechte von Unternehmen am geistigen Eigentum werden bis zu einem unbestimmten Grad ausgeweitet.

$\sqcup$ Der Zugang zu Information und Wissen hängt von der Kaufkraft ab.

- Die Machtkonzentration auf den Informations- und Wissensmärkten wird nur geringfügig korrigiert.

$\square$ Es gibt keine umwelt- oder arbeitsrechtlichen Einschränkungen, die der Entwicklung und der Anwendung von Informations- und Kommunikationstechnologien Grenzen setzen könnten.

$\checkmark$ Zahlreiche Menschen wissen mehr über Konsumgewohnheiten als über demokratische Grundsätze. 
$\square$ Die Unternehmen, welche die Mehrheit der technologischen Ressourcen und Inhalte der Informationsgesellschaft kontrollieren, sind gegenüber der Öffentlichkeit nur am Rande rechenschaftspflichtig.

$\checkmark$ Bei der Entwicklung von IKT ist die Rentabilität ein wichtigerer Faktor als die menschliche Sicherheit.

$\square$ Der Öffentlichkeitsbereich wird so weit als möglich eingeschränkt.

Um den WSIS-Prozess so zu beeinflussen, dass eine auf Bürgerbeteiligung und Menschenrechte beruhende Vision der Informationsgesellschaft entsteht, müsste ein Forum geschaffen werden, das sich für die Errichtung einer Informationsgesellschaft von der Basis her einsetzt. Potenzielle Mitglieder eines solchen Forums sind progressive Organisationen der Zivilgesellschaft, kritische Vertreter der Kommunikations- und Informationswissenschaften, öffentliche Amtsträger mit abweichenden Ansichten, Vertreter der Industrie und Technik mit sozialem Verantwortungsbewusstsein sowie unabhängige Berufsvertreter aus den Gebieten der Medien, der Telekommunikation und der Informatik.

Dieses Forum müsste zunächst eine grundlegende soziale Analyse vornehmen, welche die Hindernisse für die Umsetzung der an sich lobenswerten Visionen aufzeigt. In einem zweiten Schritt müsste öffentlicher Druck auf die Regierungen ausgeübt werden, damit sie diese Hindernisse angehen. Drittens müsste dieses Forum sich weigern, von den Regierungen in Mechanismen eingebunden zu werden, die mehrheitlich zur Legitimierung von Lösungsansätzen dienen, welche den Interessen der Mehrheit der Weltbevölkerung widersprechen. Viertens müsste sich das Forum massiv in Bildungsprogrammen und Mobilisierungskampagnen engagieren und in der breiten Öffentlichkeit Aufmerksamkeit wecken, damit informations- und kommunikationsrelevante Themen in den gesellschaftspolitischen Debatten aufgegriffen werden. Fünftens müsste das Forum Beispiele für eine Gute Praxis entwickeln, welche die Bürgerbeteiligung in den Bereichen Medien, Telekommunikation und Informationstechnologie gewährleisten. Sechstens müsste auf der Basis der grundlegenden völkerrechtlichen Bestimmungen im Bereich der Menschenrechte eine politische Agenda erarbeitet werden. Siebtens muss dieses Forum aufzeigen, dass das geltende System zum Schutz der Rechte am geistigen Eigentum die Plünderung der genetischen Ressourcen in den armen Ländern (biopiracy) rechtfertigt und die Interessen der Unternehmen besser schützt als jene lokaler Gemeinschaften oder einzelner Künstler. Zentrale Elemente dieses Forums müssen der Schutz des Allgemeingutes und der kulturellen Ressourcen sowie der Schutz der öffentlichen Güter vor der Nutzung durch private Unternehmen sein.

Im Bereich der Menschenrechte muss darauf abgezielt werden, die Kultur von handelsrechtlichen Bestimmungen zu befreien und einen Spielraum für nationale Massnahmen zum Schutz der kulturellen Eigenständigkeit und des lokalen Gemeinguts zu schaffen. Die heutigen globalen Unternehmenszusammenschlüsse wirken sich sowohl für Konsumenten als auch für Berufstätige negativ aus, da sie zu einer Verringerung der Vielfalt und zu einem Verlust der beruflichen Autonomie führen. Eine Menschenrechtsagenda soll sicherstellen, dass das gemeinsame menschliche Erbe auch in Zukunft ein öffentliches Eigentum 
ist, so dass die öffentliche Rechenschaftspflicht und die gemeinschaftlichen Ansprüche gewährleistet bleiben.

Zudem müsste das Forum darauf hinarbeiten, dass der Schlussdeklaration und dem Aktionsplan des WSIS - im Namen der unterzeichnenden Regierungen folgende Präambel vorausgeht:

- Wir sind der Überzeugung, dass die gegenwärtigen weltweiten wirtschaftlichen Beziehungen höchst unfair sind und der Mehrheit der Weltbevölkerung Nachteile bringen.

- Wir sind der Überzeugung, dass der gegenwärtige wirtschaftliche Globalisierungsprozess die Nutzen und Risiken äusserst ungleich auf der Welt und innerhalb der einzelnen Gesellschaften verteilt.

- Wir sind überzeugt, dass die Welthandelsorganisation und ihre Politiken grundlegend reformiert werden müssen, damit sie die Interessen der Weltbevölkerung auf gerechtere Weise wahrnehmen kann.

- Wir sind der Überzeugung, dass das System der Vereinten Nationen reformiert werden muss, damit die Grundsätze der demokratischen Beteiligung und der Verantwortung gegenüber der Öffentlichkeit gewährleistet sind.

$\square$ Wir sind der Überzeugung, dass alle Länder, welche Massenvernichtungswaffen besitzen, entwaffnet werden müssen.

$\sqcup$ Wir werden nicht länger akzeptieren, dass jeden Tag 30'000 Kinder unnötigerweise verhungern oder wegen anderer vermeidbarer Mängel der heutigen Weltordnung sterben müssen.

$\square$ Wir setzen uns ein für eine gerechtere Verteilung der Ressourcen auf der Welt, und als Vertreter wohlhabender Nationen verpflichten wir uns, alles in unserer Macht stehende zu unternehmen, um die Bevölkerungen unserer Länder zu überzeugen, ihren auf Konsum ausgerichteten Lebensstil zu ändern.

$\checkmark$ Wir verpflichten uns, den Internationalen Strafgerichtshof in Den Haag in seiner Tätigkeit nach Kräften zu unterstützen.

$\square$ Wir verpflichten uns zur Einhaltung aller Menschenrechte, seien sie bürgerlicher, politischer, gesellschaftlicher, wirtschaftlicher oder kultureller Natur, und zur Schaffung wirksamerer Mechanismen für deren Verteidigung.

- Wir sind der Überzeugung, dass das in den vergangenen Jahren ernsthaft unterminierte gesellschaftliche Vertrauen die Basis bildet für jede demokratische Einigung, und dass den vielfältigen Formen der Enttäuschung, der Lüge und Beeinflussung, deren sich die Regierungen unentwegt bedienen, Einhalt geboten werden muss.

」 Wir verpflichten uns, sämtliche völkerrechtlichen Vereinbarungen im Zusammenhang mit der Informationsgesellschaft, die derzeit in Kraft sind oder verhandelt werden, zu überprüfen, zu überarbeiten und ihnen beizutreten, so dass sie mit den im Völkerrecht verankerten Menschenrechten umfassend vereinbar sind. 
Natürlich klingt dies alles äusserst romantisch und unrealistisch. Aber ohne ein ernsthaftes Engagement in diesen Bereichen haben die wohlmeinenden Aussagen des WSIS für die meisten Menschen dieser Welt keine Bedeutung.

\section{Quellen}

ITU, World Telecommunication Development Report, ITU, Genf, 1997;

ITU, General Trends in Telecommunication Reform, 1998 World, volume I, ITU, Genf, 1998 ;

Maitland, D., The Missing Link: World Telecommunications Forum Report, ITU, Genf, 1986;

Rorty, R., Putting your money where your mouth is, UNESCO Sources No. 79/May, UNESCO Press, Paris, 1996;

UNDP, Human Development Report 1998, Oxford University Press, Oxford, 1998 ;

Venturelli, S. „Cultural Rights and World Trade Agreements in the Information Society“, Gazette 60.1: 47-76, 1998; 